\title{
Integrated College Bus Tracking System
}

\author{
J. Navya Sree ${ }^{1}$, C. Mounika ${ }^{1}$, T. Mamatha ${ }^{1}$, B. Sreekanth ${ }^{1}$, N. Diwakar ${ }^{1}$, Noor Mohammed ${ }^{2}$ \\ 1B.TECH Students, Department of Computer Science and Engineering, Sanskrithi School of Engineering, \\ Puttaparthi, Andhra Pradesh, India \\ ${ }^{2}$ Assistant Professor, Department of Computer Science and Engineering, Sanskrithi School of Engineering \\ Puttaparthi, Andhra Pradesh, India
}

\begin{abstract}
Article Info

Volume 8, Issue 3

Page Number : 732-735

\section{Publication Issue}

May-June-2021

\section{Article History}

Accepted : 10 June 2021

This paper proposes a smart application for College Bus Tracking System, which runs on Android smart phone. This enables students to find out the location of the bus so that they will not get late or will not arrive at the stop too early. The main purpose of this application is to provide exact location of the student's respective buses and providing information like bus details etc. This application may be widely used by the college students since Android smart phones have become common and affordable for all. It is a real time system as the current location of the bus is updated every moment in the form of latitude and longitude, which is received by the students.
\end{abstract}

Published : 17 June 2021
Keywords : GPS, Tracking system.

\section{INTRODUCTION}

The mobile phone industry is one of the fastest and most dynamic business sectors today. The need to communicate efficiently and instantaneously is always an undying necessity. The market sector and the ever-growing and demanding consumers always want to have more, and they want it better than ever. Having a mobile phone for us makes life easier. Communication is always a part of daily life, and we cannot avoid it. The invention of mobile phone has gone tremendous leaps in innovation and new applications. Originally, it was intended to be a telephone that can be carried wirelessly at greater distances. Advances in communications, upgrades in radio frequency and developments on the internet had given mobile phones more sophisticated but easy to use in applications.

Mobile phones are now equipped with navigation systems such as GPS or Global Position Satellite that aids travelers, tourists, adventurers and navigators on getting the most accurate view on their present location. Not only that, it also assists them in searching for the nearest establishment that they want to look for such as restaurants, hotels, malls, bank, police station and other places of interests.

A vehicle tracking system is one of the most common applications used for tracking vehicles which is also used to prevent vehicle from theft. Today Android 
Applications are very good source for tracking the vehicles. It provides real time data on the movement of vehicles. Android phones are widely used for this purpose because they have GPS device attached with it. It acts as both transmitter as well as receiver. A vehicle tracking system combines the use of automatic vehicle location in individual vehicles with software that collects these fleet data for a comprehensive picture of vehicle locations. Modern vehicle tracking systems commonly use GPS for locating the vehicle, but other types of automatic vehicle location technology can also be used. Vehicle information can be viewed on electronic maps via the Internet. Urban public transit authorities are an increasingly common user of vehicle tracking systems, particularly in large cities.

\section{LITERATURE REVIEW}

[1] Priyanka V. Narkhedeal, "Bus Tracking System based on Location-Aware Services", International Journal of Emerging Technologies in Engineering Research, Volume 6, Issue 3, March 2018.

In day-to-day life, people travel from one place to another and most of the population use Bus as a medium to reach their destination. This paper mainly focuses on the problem with the buses, that the passengers do not know the exact timing of the arrival of buses. The location of the bus and routes taken by the buses could be easily tracked on a smartphone. Global Positioning System and Google Maps are used for navigation. An application based on the android is used, which includes information of all routes and bus details. The application is updated occasionally so that all the changes in bus routes and timings are noted.

The user could request for the location of the bus, and the details stored in the database via a GPS device fitted on the bus can be retrieved whenever needed.

[2] PAPER2.International Research journal of Engineering and Technology (IRJET), Sep Real Time College Bus Monitoring and
Notification System M. S. Minu, Deepak Adithya K. N. In today economic and traffic condition no one can predicts at what time and when the required transportation of a person can arrive .The aim of the paper work to provide a app which can be used for college students so that they can manage the time during all days usefully and get to their transport point at the right time and not lose the bus or any other college transportation receive provided by the college.

[3] Shubham Jain et al., "Application-based bus tracking system", 2019 International Conference on Machine Learning, Big Cloud and Parallel Computing, 1416 Feb 2019.

This paper is based on a bus tracking system, in which a GPS Tracking application is used to track the bus. The passengers are unaware of the information regarding bus timing and therefore waste their time waiting for the bus on their particular route. GPS technology is user-oriented, to receive the navigating instructions at any instant of time. Here, the location of the bus is received from the satellite and then with the help of cellular networks, it is further processed and sent to the web-server. The coordinates received are processed through Google Maps API. Google Maps API helps to collect data like latitudes and longitudes, locations, etc.

The data received is processed in the user's device, to display the real-time information.

[4] Sharmin Akter et al., "A Cloud- Based Bus Tracking System based on Internet of Things Technology", 2019, 7th International Conference on Mechatronics.

In this paper, a Cloud-based bus tracking system based on IoT is proposed. The combination of cloud computing and the Internet of Things enables monitoring the bus services, which need to be stored, processed, and assessed. This paper proposed a mobile application, through which the mainly focuses on the problem with the buses, that the passengers do not know the exact timing of the arrival of buses. The location of the bus and routes taken by the buses 
could be easily tracked on a smartphone. Global Positioning System and Google Maps are used for navigation. An application based on the android is used, which includes information of all routes and bus details. The application is updated occasionally so that all the changes in bus routes and timings are noted. The user could merely request for the location of the bus, and the details stored in the database via a GPS device fitted on the bus can be retrieved whenever needed.

\section{PROPOSED SYSTEM}

The proposed system provides the exact location of our college bus to the students and staff from their location through mobile application. In this project to receive the signal from the satellite, the driver should turn on the GPS in his Android smartphone. The device receives the GPS data and sends the latitude and longitude values of the location of the bus to the server at regular intervals.

\section{ADVANTAGES OF PROPOSED SYSTEM}

-Makes students to avoid unexpected delays.

-Real time monitoring of college buses.

\section{ARCHITECTURE OF PROPOSED SYSTEM}

Authorized person passengers can track the location of the bus and estimate the arrival time of the bus. It is also provided with the nearest stoppage from the current location. Therefore, passengers with no worry can utilize their waiting time by choosing the nearest route.

[5] Priyanka V. Narkhede et al., "Bus Tracking System based on Location-Aware Services", International Journal of Emerging Technologies in Engineering Research, Volume 6, Issue 3, March 2018.

\section{GPS SIGNAL}

GPS sends position data to server

\section{SERVER}

REQUEST BUS ARRIVAL
In day-to-day life, people travel from one place to another and most of the population use Bus as a medium to reach their destination. This paper

\section{ANDROID APP FOR MOBILE USER MODULES}

\section{Driver 2.Student 3.Admin}

Driver Module: This module is designed for the bus driver. The authorized bus drivers can use this module by providing their unique login credentials. They need to start their location services before driving. The current location of the bus will automatically be updated from the driver's mobile to the server every moment.

Admin Module: This module is designed for the bus administrator for updating the information. Admin can $\log$ in to the admin account after authentication and authorization. He can add the driver, buses and students.

Student Module: This is the most important module and the soul of the system. Users of this module need to click on the student login. They can access the details of all the buses through their smartphones. Here, they will get all the buses information. Students can track the location of their buses from any location. A student must make sure that their location service is active.

\section{SNAPSHOTS}

Login page

\section{v. CONCLUSION AND FUTURE SCOPE}

In this project we designed and developed a real time bus tracking system using Android studio. In this project we try to save the time of the students mainly who uses the college transportation service and we also tend to help them with easy and tension mornings for a bright and peaceful day. This application does not need any external hardware except a smartphone which is available to all the students. So, the overall cost is very low or no cost needed for tracking the bus location. It provides 
nearly accurate data in real time that makes possible for the user to track the buses.

The proposed system can further be enhanced by making use of it in college bus tracking systems and also to report accidents and help in traffic monitoring. It can also be made compatible with IOS and Windows OS supporting systems.

\section{REFERENCES}

[1]. P. Chaiprapa, S. Kiattisin, and A. Leelasantitham, "A Real- Time GPS Vehicle Tracking System Displayed on a Google-Map- Based Website," University of the Thai Chamber of Commerce Journal, 2011.

[2]. P. Zhou, Y. Zheng, and M. Li, "How Long to Wait? Predicting Bus Arrival Time with Mobile Phone Based Participatory Sensing, "Transactions S. A. E. Yosif, M. M. Abdelwahab, M. A. E. ALagab, and F. Muhammad, "Design of Bus Tracking and Fuel Monitoring System,"In Proc. of International Conference on Communication, Control,Computing and Electronics Engineering (ICCCCEE), IEEE,Khartoum, Sudan, 16-18 January 2017.

[3]. Kumbhar, Meghana Survase, Pratibha Mastud, Avdhut Salunke, "Real Time Web Based Bus Tracking System," International Research Journal of Engineering and Technology (IRJET), Volume: 03 Issue: 02, Feb2016.

[4]. Sharmin Akter, Thouhedul Islam, Rashidah F. Olanrewaju, Ajayi Adeniyi, "A Cloud- Based Bus Tracking System based on Internet of Things Technology," 7th International Conference on Mechatronics, 2019.

[5]. Priyanka V. Narkhede, Radhika V. Mahalle, Priya A. Lokhande, Reetu M. Mundane, Dhiraj M. Londe, "Bus Tracking System based on Location-Aware Services," International Journal of Emerging Technologies in Engineering Research, Volume 6, Issue 3, March 2018.
[6]. Benjamin Y.O. Low, Samsul Haimi Dahlan, Mohd Helmy Abd Wahab, "Real-time Bus Location and Arrival Information system," IEEE Conference on Wireless Sensors (ICWiSe), 2016.

\section{Cite this article as :}

J. Navya Sree, C. Mounika1, T. Mamatha, B. Sreekanth, N. Diwakar1, Noor Mohammed, "Integrated College Bus Tracking System", International Journal of Scientific Research in Science and Technology (IJSRST), Online ISSN : 2395-602X, Print ISSN : 2395-6011, Volume 8 Issue 3, pp. 732-735, May-June 2021. Available at doi : https://doi.org/10.32628/IJSRST2183164 Journal URL : https://ijsrst.com/IJSRST2183164 